\title{
LA PARTICIPACIÓN DE LA POBLACIÓN EN LA ACTIVIDAD ECONÓMICA
}

\author{
BRígIdA García \\ El Colegio de México
}

\section{INTRODUCCIÓN}

EL PRESENTE artículo tiene por objeto analizar los niveles de participación de la población mexicana en la actividad económica durante el período $1950-1970 .^{1}$ Como es conocido, la información censal para este período muestra un descenso de gran proporción en dichos niveles, cuyo origen no ha sido estudiado lo suficiente. En el trabajo se analiza la influencia de tres diferentes factores sobre el descenso de los niveles de participación: 1) el crecimiento de la población y el consiguiente cambio en la composición por sexo y edad; 2) la comparabilidad de la información censal; y 3) el crecimiento económico experimentado en el país en el período bajo estudio, observado a través de los cambios en la estructura ocupacional. Esta separación se hace sólo con fines analíticos, ya que se es consciente de la interrelación existente entre los tres diferentes factores, lo cual se comprueba en el desarrollo del trabajo.

\section{Descripción de los NiVELes. El EFECTO DE LOS FACTORES DEMOGRÁFICOS}

\section{La tasa bruta de actividad}

El indicador más sencillo que muestra la relación existente entre el tamaño de la población económicamente activa (PEA) y el de la población total (PT) es la llamada tasa bruta de actividad $\left(T B A=\frac{P E A}{P T} \times 100\right)$.

* Agradezco a Mercedes Pedrero, Gustavo Garza y Sofía Méndez sus valiosos comentarios a una primera versión, más amplia, de este trabajo.

1 La elección del período responde más bien a problemas de orden censal, la información recolectada sobre población económicamente activa sólo ofrece un mínimo de comparabilidad en el caso de los últimos tres levantamientos (véase el apéndice 1 de la versión ampliada de este trabajo, en mimeógrafo). Se entiende por población económicamente activa (PEA) aquella parte de la población que se encuentra disponible para la producción de bienes y servicios de índole económica en un período determinado. 
Se denomina tasa bruta porque relaciona la población activa con el volumen total de la población, incluyendo aquí a las personas cuya edad las incapacita para participar en la población activa. ${ }^{2}$

Las TBA por sexos para 1950-1970 se presentan en el cuadro 1 en el cual se observan dos hechos importantes: 1) tanto las tasas globales, como las de los dos sexos descienden, o tienden a descender sistemáticamente durante el período; 2) las tasas para 1970 se apartan considerablemente de las tendencias que muestran los datos de los años anteriores; en el caso de los hombres el descenso es mucho más brusco en el segundo decenio que en el primero, $y$ en el caso de las mujeres la tendencia ascendente se invierte durante este último decenio.

Cuadro 1

MÉxico: TASAS BRUtas DE ACTIVIDAd POR SEXos, 1950-1970 (Porcientos)

\begin{tabular}{lccc}
\hline Lño & Total & Howbres & Mujeres \\
\hline 1950 & 32.3 & 56.8 & 3.7 \\
1960 & 32.2 & 53.0 & 11.5 \\
$1970^{a}$ & 26.8 & 43.6 & 10.2 \\
\hline
\end{tabular}

Fuentes: VII, VIII y IX Censos Generales de Población.

a PEA del año de 1969.

En condiciones de pleno empleo y de productividad del trabajo constante, un descenso en la TBA significaría un descenso en el nivel de ingreso per capita. Ya que tales condiciones no se dan en un país como México, habría que interpretar el descenso de la TBA como un aumento en el grado de dependencia de la población inactiva respecto a la activa. Este aumento fue mucho más pronunciado en el decenio 1960-1970, ya que el descenso porcentual de la TBA total en este decenio fue de $16.8 \%$ en comparación con un descenso de $0.31 \%$ en el anterior $(17.03 \%$ para el período completo). Este hecho parecería confirmar la posición neomalthusiana sobre el papel que juega la población en el desarrollo del país; sin embargo, la realidad es mucho más compleja de lo que a primera vista parecen reflejar estas cifras. El crecimiento de la población sin duda contribuyó al descenso de la tasa - su efecto será cuantificado más adelante-; no obstante, existen otros dos factores cuya influencia debe ser analizada, al menos preliminarmente, en este momento.

a) La influencia del desarrollo económico sobre los niveles de participación. En los estudios comparativos sobre niveles de participación en países desarrollados y subdesarrollados, comúnmente se considera que el descenso en la participación masculina es más bien un indicador de desarrollo económico, ya que generalmente se ha encontrado una rela-

2 Por lo general, esta edad se fija arbitrariamente. En el caso de México, sólo los mayores de 12 años pueden formar parte de la PEA (véase el apéndice 1 de la versión ampliada). Este es un critcrio estrictamente censal; en realidad no se afirma que no existan trabajadores menores de 12 años. 
ción inversa entre dicho nivel y el nivel de desarrollo.3 Se explica que el descenso es una respuesta a las demandas de calificación y eficiencia creadas por la sociedad industrial moderna. Por una parte se argumenta que los jóvenes necesitan mavor tiempo de entrenamiento y por lo tanto postergan su entrada a la actividad; por otra, se afirma que a medida que se consolidan los sistemas de seguridad social públicos y privados los de mayor edad se retiran cada vez más pronto. Éste es un planteamiento quizás demasiado simplista, ya que por lo general sólo toma en consideración los cambios en la participación en las edades extremas. Habría que analizar en qué medida esta situación corresponde al caso mexicano, a fin de poder considerar el descenso en la participación masculina como un indicador de desarrollo económico (este análisis se realiza en la última sección).

b) Comparabilidad de la información censal. Los problemas que presenta la información pueden ser al menos detectados a través de un análisis preliminar de los niveles de participación femeninos. Al contrario de lo que sucede en otras regiones del mundo subdesarrollado, en especial el sudeste asiático y el sur de África, la participación de la mujer latinoamericana en la actividad económica ha sido tradicionalmente baja. ${ }^{4}$ Por lo general, esta situación ha variado poco en las regiones agrícolas latinoamericanas; en cambio, en las ciudades, ya para principios del decenio de los sesenta, entre el 25 y el $33 \%$ de las mujeres adultas tenía algún tipo de trabajo (en el servicio doméstico y personal especialmente). Estas eran tasas similares a las que presentaban entonces los países industrializados. $^{5}$

México parece haber participado de este aumento en la participación femenina, principalmente en las actividades económicas urbanas, durante el decenio 1950-1960, pero en apariencia la tendencia no se mantuvo en el decenio siguiente. Este último descenso, un factor que sin duda influye en forma importante en la baja de la participación total, es bastante difícil de explicar en vista del incremento anterior. El ritmo de expansión económica no cambió sustancialmente de un período a otro; el aumento en el nivel de escolaridad y el incremento de la urbanización, factores considerados en el primer decenio como parcialmente explicativos del cambio, ${ }^{6}$ se ha sostenido, aunque no habría que olvidar que

3 Por lo general, en estos estudios se mide el nivel de desarrollo económico a través de indicadores como el producto per capita, el porciento de trabajadores agrícolas o manuales, población urbana, consumo de energía, etc. Véase, por ejemplo, John D. Durand, "Tasas de actividad y desarrollo económico en América Latina", Conferencia Regional Latinoamericana de Población, Actas 2, México, El Colegio de México, 1970, pp. 77-82. Durand analiza aquí los cambios en los niveles de participación de 19 países americanos durante los períodos 1946-1953 y 1960-1964.

4 Véase, Esther Boserup, Women's Role in Economic Development, Londres George Allen and Unwin, 1970, pp. 174-191.

s Ibid., p. 187.

6 Véase Mercedes Pedrero Nieto, Labour Force in Mexico: a Study of Regional Variations, Tesis doctoral inédita, p. 23. 
la rapidez del proceso de urbanización ha comenzado a disminuir en los últimos años. ${ }^{7}$

Esta aparente paradoja, y otras que surgen a lo largo del trabajo, deben interpretarse con cuidado en vista de los cambios en las definiciones censales que se utilizaron en 1970. Este problema se analiza con detalle en la segunda sección.

\section{La tasa refinada de actividad}

La tasa refinada $\left(T R A=\frac{P E A}{P T(12 \text { años y más })} \times 100\right)$ es un indicador de participación que relaciona la PEA con la población verdaderamente expuesta al riesgo de participar. Al comparar los resultados que presenta este indicador con los de la TBA se puede precisar el efecto del crecimiento de la población menor de 12 años sobre las tendencias antes analizadas. Conviene aclarar de antemano que en países como México, de alta natalidad y baja mortalidad, la proporción que los menores de 12 años representan respecto de la población total tiende a crecer $(34.5 \%$ de la población en 1950; 36.9 en 1960 y 38.4 en 1970). En el cuadro 2 se presentan las tasas refinadas por sexo para el período 1950-1970.

Cuadro 2

MÉxico: TASAS REFINADAS DE ACTIVIDAd POR SEXos, 1950-1970 (Porcientos)

\begin{tabular}{cccc}
\hline Lño & Total & Howbres & Yujeres \\
\hline 1950 & 49.4 & 88.0 & 13.1 \\
1960 & 51.0 & 85.1 & 18.0 \\
1970 & 43.6 & 71.7 & 16.4 \\
\hline
\end{tabular}

Fuente: VII, VIII y IX Censos Generales de Población.

A diferencia de la TBA, la TRA total aumenta entre 1950 y 1960 para descender luego en el siguiente decenio hasta alcanzar en 1970 un nivel más bajo que el de 1950 (un descenso de $11.7 \%$ para el período completo). Si analizamos el fenómeno tomando cada sexo por separado, se observa que en el caso de los hombres, la TRA desciende sistemáticamente al igual que la TBA pero de manera más lenta (en el decenio 1950-1960 el descenso en la TRA fue de 3.3\% y el de la TBA de $6.7 \%$; durante 1960-1970, el de la TRA fue de $15.7 \%$ y el de la TBA de $17.7 \%$; véanse los cuadros 1 y 2 ). Este descenso más lento es el resultado directo de la exclusión de la población menor de 12 años en la TRA. Al suprimir una proporción creciente de personas del denominador de la TBA masculina, la cual es una relación que por lógica decrece, se obtiene una disminución en el porcentaje de descenso. p. 121.

7. Véase, Dinámica de la población de México, El Colegio de México, 1970, 
Para las mujeres, también al igual que para la TBA, se observa un aumento en la TRA en el primer decenio y un descenso en el segundo. Aquí, sin embargo, el aumento de la TRA durante 1950-1960 fue mayor que el de la TBA (27.2\% de aumento para la TRA y 24.3 para la TBA). Por el contrario, durante 1960-1970 la TRA femenina desciende, pero el nivel de participación para 1970 se mantiene más alto que el de 1950. En resumen, durante los dos decenios se registra entonces un aumento de $21.1 \%$ para la TRA femenina, que es de todas maneras mayor que el de la твA, que fue de $14.7 \%$. Razonando de la misma manera que para el caso de los hombres, se llega a la conclusión de que al eliminar una proporción creciente de personas (las menores de doce años) del denominador de una relación que también crece (la TBA femenina para el conjunto de los 20 años) su efecto es incrementar la proporción del aumento. Por lo tanto, el crecimiento de la población menor de doce años acelera la tendencia descendente de la participación masculina, atenúa el pequeño aumento que tiende a darse en la femenina y, por lo tanto, acelera el descenso total. De manera más precisa, este crecimiento de la población menor de doce años explica el $31.06 \%$ del descenso de los niveles de participación durante el período. ${ }^{8}$ Durante el primer decenio, dicho crecimiento explica el descenso que muestra la tasa bruta (si se elimina su efecto, el nivel de participación aumenta). Sin embargo, en el segundo decenio se mantiene la tendencia descendente aun si no se toman en cuenta a los menores de doce años. Esto sugiere, como veremos a continuación, que muchos otros factores, aún no analizados, inciden de manera importante sobre los niveles de participación.

\section{La tipificación}

Al considerar el crecimiento de la población menor de doce años no hemos aislado totalmente la influencia de los factores demográficos sobre los niveles de participación. En países como México la estructura por edades de la población mayor de doce años también sufre variaciones importantes. La tipificación de la TRA precisará la medida en la que este indicador desciende debido a variaciones en dicha estructura, y la medida en que lo hace debido a variaciones en la participación económica en los distintos grupos de edad. Sin embargo, los dos efectos no actúan completamente aislados como comprobaremos más adelante.

La información necesaria para el cálculo de la tipificación se presenta para hombres y mujeres por separado en los cuadros 3 y 4 . En las columnas 2, 3 y 4 aparece la estructura por edad de los mayores de 12 años para 1950, 1960 y 1970, la cual es un primer factor cuya influencia sobre las variaciones de la TRA queremos aislar.

En las columnas 5, 6 y 7 aparecen, en primer lugar, las tasas refi-

8 Efectos del crecimiento de los menores de 12 años:

$$
=\frac{11.74 \text { (descenso porcentual de la TRA) }}{17.03 \text { (descenso porcentual de la TBA) }}-1
$$




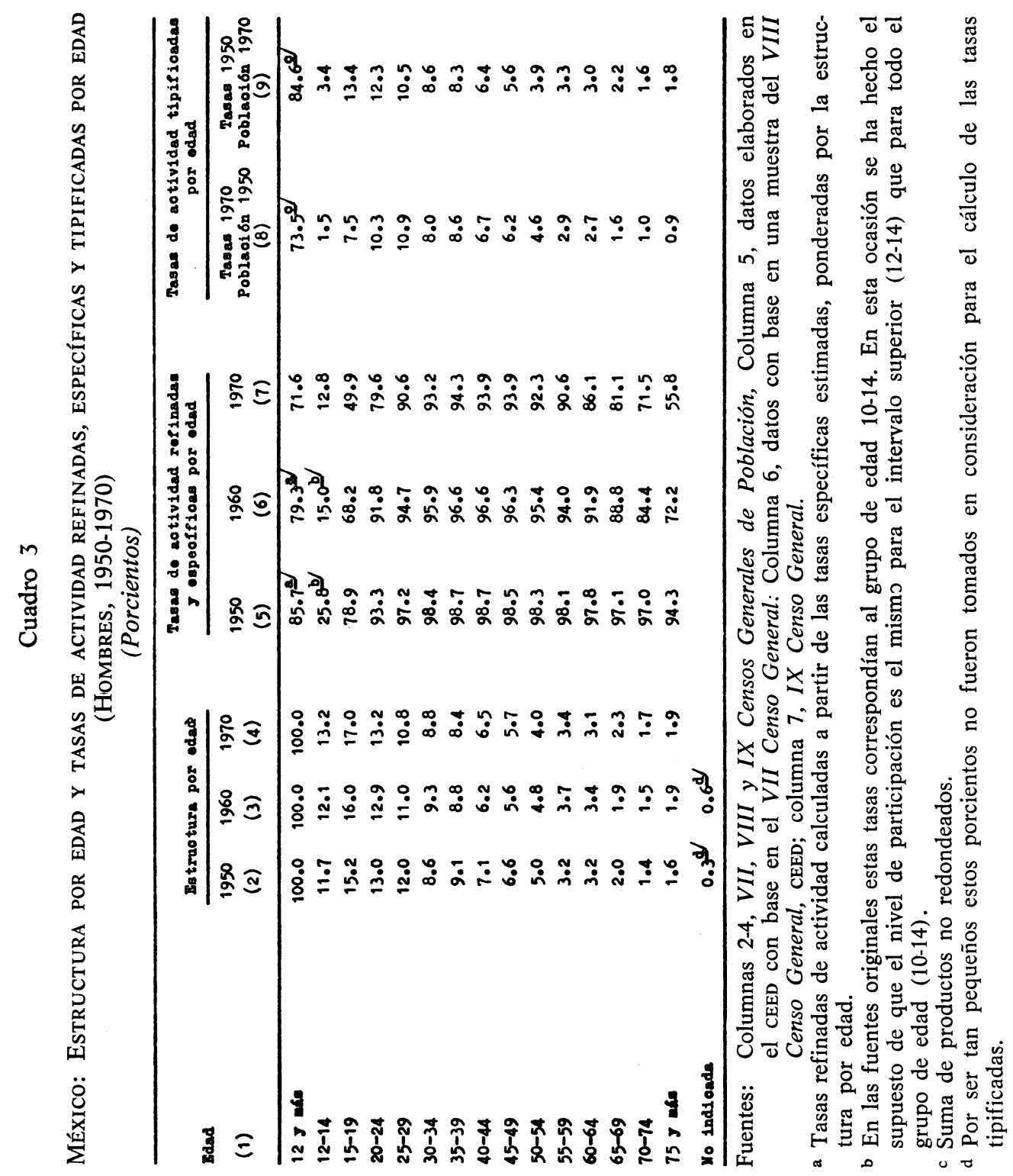




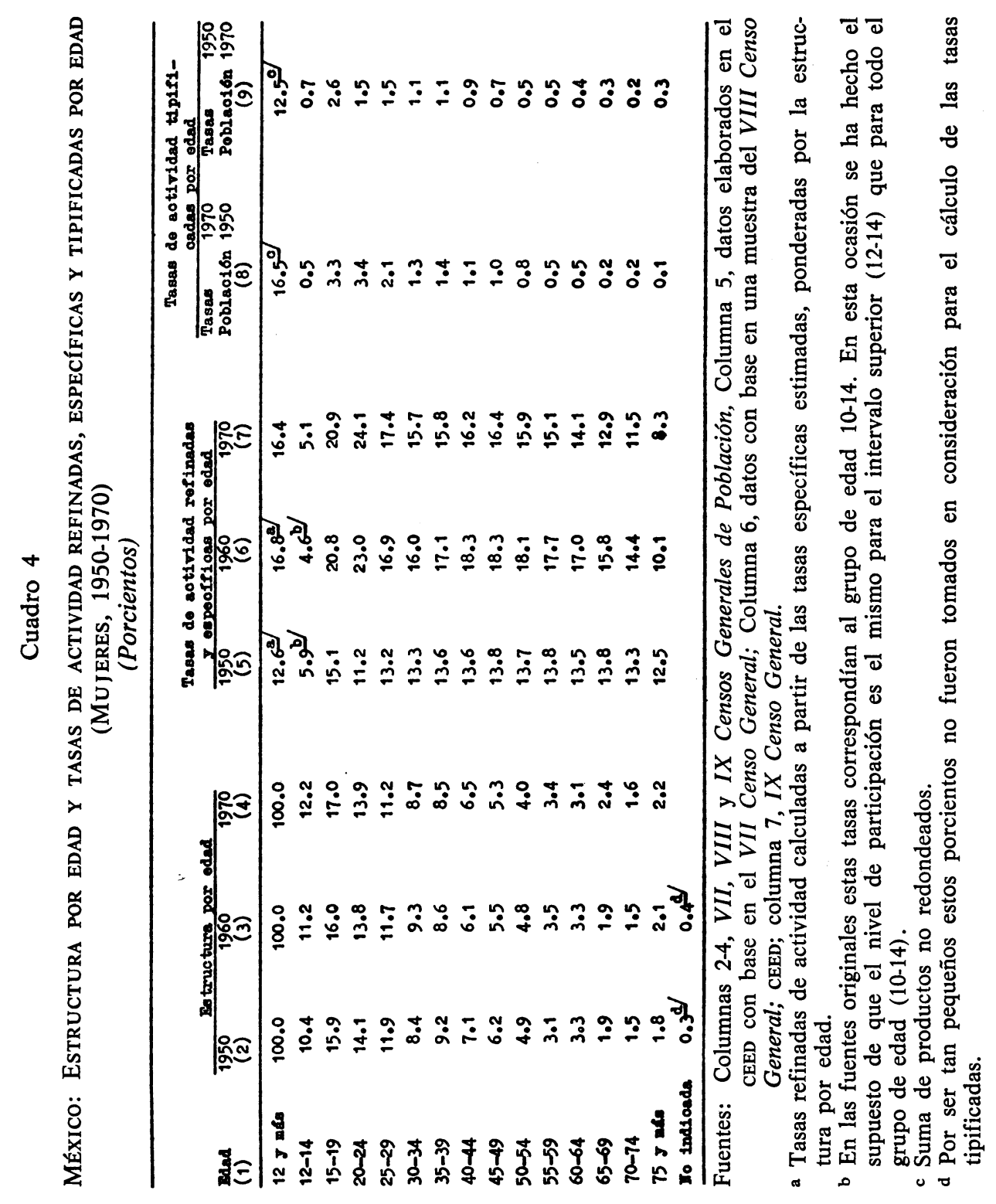


nadas (en el primer renglón las correspondientes al grupo de edad de más de 12 años), y en segundo lugar las tasas específicas de actividad por edad (en los renglones siguientes) :

$$
\begin{aligned}
& A_{x}=\frac{P E A_{x}}{P T_{x}} 100, \text { para edades individuales, } \mathrm{y} \\
& A_{x, x+4}=\frac{P E A_{(x, x+4)}}{P T_{(x, x+4)}} \times 100 \text { para grupos quinquenales de edad) } .^{9}
\end{aligned}
$$

Las tasas específicas miden el nivel de participación en los distintos grupos de edad, el cual es un segundo factor cuya influencia sobre las variaciones de la TRA queremos precisar.

Como podrá observarse, las tasas correspondientes al grupo de edad de 12 y más años en las columnas 5, 6, 7, o sea, las tasas refinadas de actividad, no corresponden a las presentadas en el cuadro 2 para los años de 1950 y 1960 . Esto se debe a que aquí la tasa refinada se expresa como un índice resumen de las tasas específicas (ponderadas por la estructura por edad) las cuales no proceden de la información censal en el caso de esos dos años. En el caso de que las tasas específicas sí procedieran de dicha fuente, no habría tal diferencia. ${ }^{10}$

9 A fin de conservar la agrupación quinquenal que más se utiliza en demografía, la primera tasa específica sólo abarca las edades 12-14, en el caso de México. La última tasa, a su vez, se expresa generalmente como un intervalo abierto a partir de los 75 años.

10 Se puede llegar fácilmente a demostrar que:

$T R A=\sum_{\mathrm{i}=1}^{14} A_{x i} \times \frac{P_{x i}}{P T(12 \text { y más años })}=\frac{P E A}{P T \text { (12 y más años })}$

Donde: TRA $=$ tasa refinada de actividad

$A_{x i}=$ tasa específica de actividad a la edad $x i$

$P_{x i}=$ población a la edad $x i$

$P T$ (12 y más años) = población de 12 y más años, $\mathrm{y}$

$\mathrm{X}_{1}=12-14, \mathrm{X}_{2}=15-19, \mathrm{X}_{3}=20-24, \mathrm{X}_{4}=25-29, \mathrm{X}_{5}=30-34, \mathrm{X}_{6}=35-39$,

$\mathrm{X}_{7}=40-44, \mathrm{X}_{8}=45-49, \mathrm{X}_{9}=50-54, \mathrm{X}_{10}=55-59, \mathrm{X}_{11}=60-64, \mathrm{X}_{12}=65-69$, $\mathrm{X}_{13}=70-74, \mathrm{X}_{14}=75$ y más.

ya que, $A_{x i}=\frac{P E A_{x i}}{P_{x i}}$, se tiene que,

$$
\begin{aligned}
& T R A=\sum_{i=1}^{14} \frac{P E A_{x i}}{P_{x i}} \times \frac{P_{x i}}{P T(12 \text { y más años })} \\
& T R A=\frac{1}{P T(12 \text { y más años })} \sum_{i=1}^{14} P E A_{x i}, \\
& \therefore T R A=\frac{P E A}{P T(12 \text { y más años })}
\end{aligned}
$$


En el apéndice 2 de la versión ampliada de este trabajo se analizan las implicaciones del uso de estas tasas específicas estimadas para los fines aquí propuestos. Allí se llega a la conclusión de que dichas tasas podrán variar ligeramente los resultados finales desde el punto de vista cuantitativo, pero no cambiar esencialmente el resultado de la tipificación que se expone después.

Si observamos ahora con detenimiento las columnas 2, 3, 4, 5, 6 y 7, en los cuadros 3 y 4, podemos notar que tanto las tasas específicas, como la estructura por edad de la población, han experimentado transformaciones durante 1950-1970. Esto sugiere que puede haber interdependencia e interacción entre los dos factores cuyo efecto buscamos aislar (tasas específicas y estructura por edad) lo cual se puede verificar mediante una tipificación que tome en cuenta este fenómeno. En este sentido, los valores de los dos factores en dos fechas determinadas, digamos principio y fin del período, pueden combinarse de cuatro maneras, conforme al siguiente esquema. ${ }^{11}$

\begin{tabular}{ccc}
\hline \multirow{2}{*}{ Estructura por edad } & \multicolumn{2}{c}{ Tasas específicas de actividad } \\
\cline { 2 - 3 } & 1950 & 1970 \\
\hline 1950 & A & C \\
1970 & D & B \\
\hline
\end{tabular}

Las combinaciones $A$ y $B$ darían las TRA no tipificadas para 1950 y 1970 respectivamente. La combinación $C$ daría la TRA de 1970 tipificada con la población de 1950 - esto es, las tasas de actividad de 1970 ponderadas por la estructura por edad de 1950- (columnas 8, cuadros 3 y 4); por último, la combinación $D$ daría la TRA de 1950 tipificada con la población de 1970, esto es, las tasas de actividad de 1950 ponderadas por la estructura por edad de 1970 (columnas 9, cuadros 3 y 4). El efecto del cambio en las tasas específicas puede ser esimado a través de la diferencia $(C-A)$ ○ $(B-D)$; los dos resultados diferirán debido a la interacción entre los cambios de los dos factores y no existe ninguna razón para preferir uno sobre el otro. Si se necesita fijar el efecto estimado del cambio en las tasas específicas en una cifra determinada, se tendrá que escoger un punto arbitrario en el campo de interacción, como veremos a continuación. De la misma manera, el efecto del cambio en la estructura por edad puede estimarse a través de la diferencia

Las tasas que pueden calcularse a partir de la información censal en 1950 y 1960 son inadecuadas para fines de la tipificación. En 1950 sólo se agrupó la PEA por grupos de edad muy amplios (12-14, 15-19, 20-24, 25-44, 45-64 y 65 y más) y no se presentó esta información clasificada por sexo. En 1960, a partir del grupo de edad 25-29, la PEA masculina en cada grupo de edad es mayor que la PEA total.

11 Para mayor información sobre este tipo de tipificación véase, Métodos para analizar datos censales sobre las actividades económicas de la población, Naciones Unidas, ST/SOA/Series A/43 pp. 43-46. 
$(D-A)$ o $(B-C)$. Conforme a lo anterior, el cambio neto $(B-A)$ de la TRA durante el período podrá entonces ser factorizado de dos maneras:

$$
\begin{aligned}
(B-A) & =(C-A)+(B-C) ; 0 \\
& =(D-A)+(B-D)
\end{aligned}
$$

Procedamos ahora a sustituir las letras por las cifras correspondientes ${ }^{12}$ tomando cada sexo por separado.

Para el caso de los hombres: $A=85.7$ (cuadro 3, columna 5), $B=71.6$ (cuadro 3, columna 7), $C=73.5$ (cuadro 3, columna 8), y $D=84.6$ (cuadro 3, columna 9). Conforme a las dos maneras de factorizar mencionadas antes tendríamos los resultados que aparecen en el cuadro 5 .

\section{Cuadro 5}

\begin{tabular}{ccc}
\hline Cambio neto & $\begin{array}{c}\text { Efecto de los cambios en } \\
\text { las tasas especfficas }\end{array}$ & $\begin{array}{c}\text { Efeoto de los cam íos on } \\
\text { la es tructura por edad }\end{array}$ \\
\hline $71.6-85.7=-14.1$ & $73.5-85.7=-12.2$ & $71.6-73.5=-1.9$ \\
\hline
\end{tabular}

Por lo tanto, el efecto de ambos factores fue el de disminuir el nivel de la TRA; sin embargo, el efecto de las tasas específicas es sustancialmente mayor que el de la estructura por edades. Dentro del campo de interacción de este factor $(-13.0$ a -12.2) se podría fijar el punto medio (-12.6) como la estimación de su efecto preciso sobre la TRA durante 1950-1970. De la misma forma, el efecto de la estructura por edades se podría fijar aproximadamente en -1.5. La tipificación masculina nos ha proporcionado un dato sumamente valioso, el cual se analizará en detalle en lo que resta del trabajo. Nos referimos al hecho de que la influencia de la estructura por edad sobre el descenso de la TRA fue mínima; en realidad ha habido un fuerte descenso en la participación económica en los distintos grupos de edad, lo cual es un fenómeno que tiene repercusiones importantes como se verá más adelante.

Para el caso de las mujeres: $A=12.6$ (cuadro 4, columna 5), $B=16.4$ (cuadro 4, columna 7 ),$C=16.5$ (cuadro 4, columna 8), y $D=12.5$ (cuadro 4 , columna 9 ).

Si se factoriza de la misma manera que en el caso anterior, se obtienen los resultados del cuadro 6.

El efecto de las tasas específicas en este caso fue el de elevar la TRA y el de la estructura por edades el de disminuirla. Sin embargo, tal y como en el caso de los hombres, el efecto de las tasas específicas

12 Aquí sólo se realiza la tipificación para los 20 años en conjunto. En el adéndice 2 de la versión ampliada ésta se lleva a cabo para los dos decenios por separado. Las conclusiones a las que se llega en ambos casos son prácticamente las mismas. 
Cuadro 6

\begin{tabular}{ccc}
\hline Cambio neto & $\begin{array}{c}\text { Efecto de los cambios en } \\
\text { las tasas especfficas }\end{array}$ & $\begin{array}{c}\text { ifecto do los cambios on } \\
\text { la es tructura por edad }\end{array}$ \\
\hline $16.5-12.6=+3.9$ & $16.4-16.5=-.1$ \\
$16.4-12.6=+3.8$ & $16.4-12.5=+3.9$ & $12.5-12.6=-.1$ \\
\hline
\end{tabular}

es mucho mayor. El efecto de la estructura por edades es tan pequeño que aquí no se presenta ninguna interacción entre los dos factores.

El uso de la tipificación y de las demás técnicas demográficas analizadas en esta sección, paradójicamente señala que los factores demográficos sólo tienen una importancia secundaria en la explicación de las tendencias de los niveles de participación. Más adelante intentaremos aproximarnos a dicha explicación analizando la comparabilidad de la información, buscando establecer relaciones entre la participación y los cambios operados en la estructura económica del país entre 1950 y 1970. Como las tasas específicas son los indicadores de participación que reciben menor influencia de la estructura por edad, entre los que hemos analizado, el análisis posterior se basa principalmente en ellas.

III. LA COMPARABILIDAD DE LOS DATOS DEL CENSO DE 1970 CON RESPECTO A LOS DE AÑOS ANTERIORES

Una explicación de las tendencias observadas podría radicar en un aspecto insuficientemente estudiado: la falta de comparabilidad de las estadísticas que se manejan. Se analiza ahora dicha posibilidad a fin de afianzar las conclusiones posteriores sobre posibles relaciones entre variaciones en la participación y cambios en la estructura económica.

En la gráfica 1 se representan las tasas de actividad específicas por sexo y edad que aparecen en los cuadros 3 y 4 (columnas 5,6 y 7). Para el caso de los hombres, la forma que adoptan las curvas es la que por lo común se encuentra en los estudios sobre comparaciones internacionales de las tasas de actividad. Dichas tasas son reducidas en las primeras edades, donde se supone que la escolaridad es alta; alcanzan un nivel máximo de los 25 a los 55 años aproximadamente y luego descienden, a medida que los hombres comienzan a retirarse de la actividad. Al igual que los otros indicadores (tasas brutas y refinadas), las tasas específicas de participación masculina muestran un descenso sistemático, mucho más pronunciado en los primeros y en los últimos grupos de edad (12-24 y 55 y más).

Por su parte, las tasas específicas de actividad femenina no presentan una tendencia definida durante 1950-1970. En países como México, donde gran parte de la población femenina que se casa abandona la actividad, es frecuente que las tasas alcancen su nivel máximo en edades previas a la realización del matrimonio. El descenso subsiguiente por lo general 


\section{Gráfica 1}

MÉXICo: TASAS ESPECÍfICAS DE ACTIVIDAD POR SEXo Y EDAD, 1950, 1960 y 1970

(\%) 100

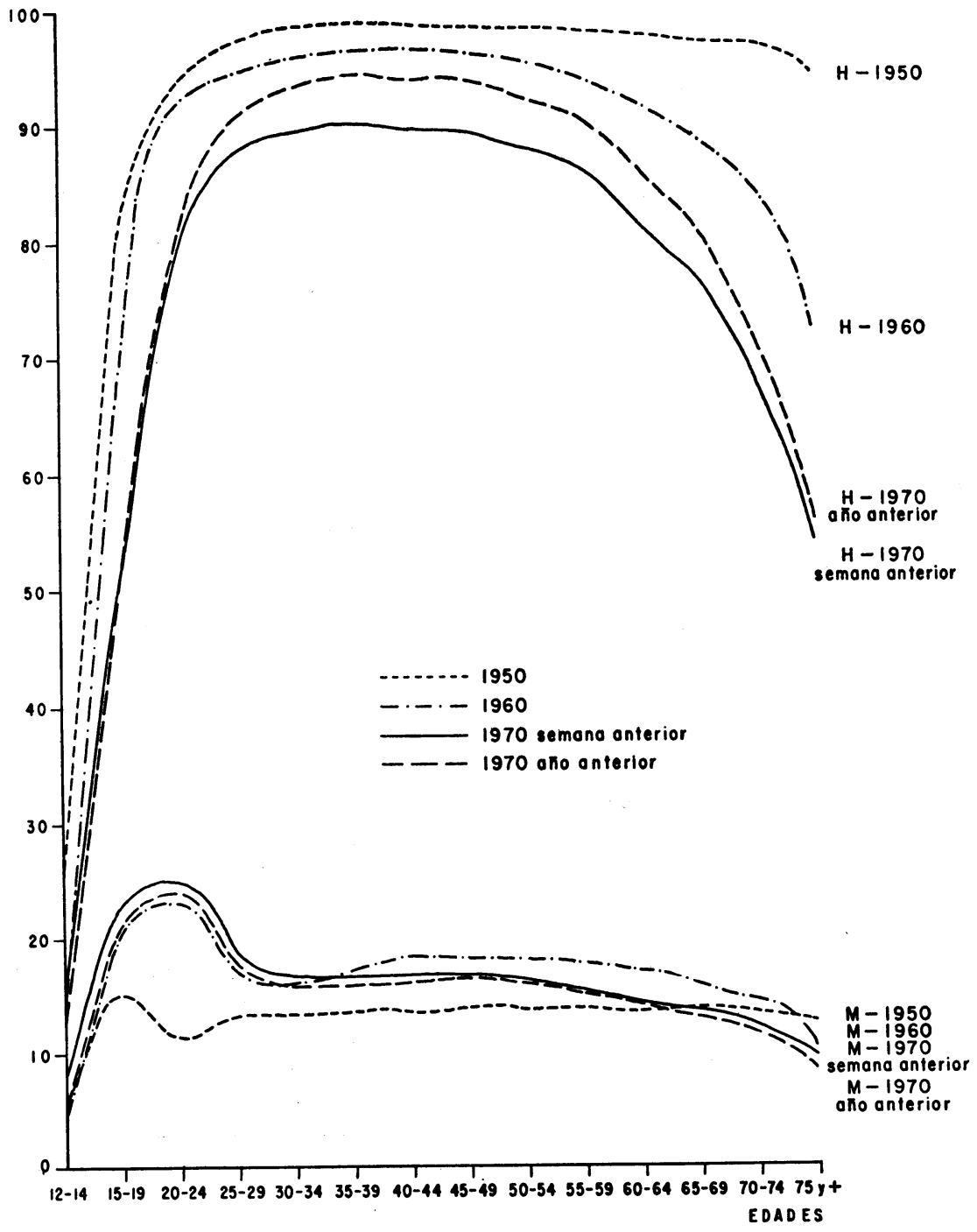

Fuente: Cuadros 3 y 4 columnas 5,6 y 7 .

es bastante brusco, aun cuando las tasas vuelven a experimentar después un aumento ligero. La interpretación usual de este fenómeno es que una pequeña parte de la población femenina casada vuelve a participar en la actividad en la última parte de su ciclo reproductivo, generalmente cuando la mayoría de los hijos han crecido. A través de la gráfica 1 puede 
observarse que la edad en la cual las tasas alcanzan su máximo nivel se ha desplazado del grupo 15-19 en 1950 al grupo 20-24 en 1960 y 1970. Esto constituiría una prueba indirecta del postergamiento de la edad al matrimonio, lo cual ha sido ya señalado en un estudio reciente sobre la nupcialidad en México. ${ }^{13} \mathrm{El}$ primer descenso que se observa en las curvas es, a su vez, mucho menos brusco en 1960 y 1970 que en el caso del año 1950. Esto aparentemente señala la permanencia de un número cada vez mayor de mujeres dentro de la actividad, aun después de casadas.

Si se analizan ahora las tendencias que presentan las tasas específicas femeninas tomando cada decenio por separado, se podrá visualizar mejor el aumento notable que mostraban los otros indicadores (tasas brutas y refinadas) en 1950-1960. ${ }^{14}$ Esto parece haber sido especialmente significativo en las primeras edades (12-14 a 20-24).

Por lo que respecta a 1960-1970, puede apreciarse a través de las tasas específicas, que el nivel de participación femenina ha descendido solamente entre las mujeres de 30 y más años. Por el contrario, la tendencia ascendente de las tasas de los primeros grupos se mantiene en el segundo decenio aunque en forma mucho menos pronunciada.

Las tendencias descritas antes deben ser interpretadas con cautela en vista de los problemas de comparabilidad que presenta la információn básica, los cuales se analizan a continuación. En la gráfica 1 se puede observar que tanto para el casu femenino como para el masculino, se han trazado dos diferentes curvas para 1970: la de la semana anterior y la del año anterior al censo. El establecimiento de períodos de referencia como los mencionados ha afectado la comparabilidad de los datos censales en numerosos países. El caso más tratado en la literatura sobre el tema es el cambio de un concepto de actividad habitual a un concepto de actividad en la semana anterior al censo.

Se afirma que: "el cambio de una pregunta de actividad usual a una actividad durante un período relativamente corto es probable que reduzca el número de personas en ciertas categorías registradas como económicamente activas: por ejemplo, aquellos que normalmente trabajan parte del año pero están inactivos en la estación en cuestión y posiblemente personas que se han jubilado o incapacitado en fecha reciente. Por otra parte, el cambio puede tener el efecto opuesto en algunas otras categorías: por ejemplo, personas que trabajan durante la estación en cuestión pero no normalmente en otras épocas del año; aquellos que trabajan ocasionalmente o irregularmente y quienes por casualidad han sido empleados o buscan trabajo durante el período en cuestión; personas de nuevo ingreso en la fuerza de trabajo que buscan sus primeros

13 Julieta Quilodrán de Aguirre, "Evolución de la nupcialidad en México, 1900-1970”, Demografía y Economía, Vol. VIII, Núm. 1, pp. 34-49.

14 Estas tasas fueron calculadas y graficadas directamente a partir de la información del cuadro 32 del "Resumen General" del IX Censo de Población. 
empleos, y posiblemente amas de casa y estudiantes que normalmente trabajan sólo por tiempo parcial. ${ }^{\mathbf{1 5}}$

La situación que presentan los datos mexicanos es en cierto modo más compleja que el simple cambio de conceptos que se expone más adelante. De hecho, la evidencia sobre la posible existencia de tiempos de referencia similares a los de 1970 , en los censos de 1950 y 1960 , es en cierto modo contradictoria. En los dos últimos se definió una persona como activa si tenía la edad necesaria y si declaraba tener una profesión $\mathrm{u}$ oficio remunerado, ya sea que lo ejerciera o no "en la fecha del censo". Esta frase ("en la fecha del censo") nunca fue suficientemente especificada, aunque en los dos casos se incluyó una pregunta sobre trabajo en la semana anterior después de las preguntas correspondientes a ocupación o profesión.

Con respecto a las tabulaciones publicadas, en el caso de 1950 existe una llamada "fuerza de trabajo por ocupación efectivamente desempeñada", cuyos totales coinciden con los del resto de tabulaciones que a primera vista parecerían más bien referirse al concepto de ocupación habitual que analizamos después. Si nos apegamos entonces a la evidencia escrita, todo parece indicar que la información publicada en 1950 fue la de la actividad desarrollada en la semana anterior al censo. Por el contrario, en 1960, no existe ninguna alusión a "ocupación efectivamente desempeñada" en las tabulaciones censales; en las instrucciones de los cuadros sólo aparece una vez la indefinida frase "en la fecha del censo". Conforme a lo anterior, sólo la información censal de 1950 y la de la semana anterior de 1970 serían estrictamente comparables desde el punto de vista de tiempos de ruferencia. No obstante, esto no asegura que de hecho lo sean desde otros puntos de vista, como veremos a continuación.

Un procedimiento indirecto que verificaría en parte el planteamiento anterior sería el análisis detallado de las variaciones en las categorías de inactivos (estudiantes, quehaceres domésticos y otros) durante el período 1950-1970, tomando a cada sexo por separado.

En 1950 y 1960 no se clasificó a ningún hombre como dedicado a los "quehaceres domésticos"; en 1970 el $2.84 \%$ de la población masculina de 12 y más años se encontraba ubicada en esta categoría (información de la semana anterior al censo). Si se analiza la distribución de estos 415920 hombres por grupos quinquenales de edad, podremos notar que constituyen por lo menos el $2 \%$ de la población masculina en todos los grupos comprendidos entre los 20 y los 59 años. Esta cifra se eleva por encima del $3 \%$ en los grupos menores de 20 años y en los mayores de $60 .{ }^{16}$ Es improbable que este tipo de inactivos haya aparecido repentinamente durante el decenio 1960-1970; más bien parecería

15 Métodos para analizar datos censales... op. cit., p. 12.

16 Información obtenida a partir del cuadro 32 del "Resumen General" del IX Censo de Población. 
que se les ha clasificado en esta categoría en virtud de algún cambio operado en el levantamiento censal.

El aumento asombroso que se registra en la categoría "otros" durante el período sería un factor adicional que debe considerarse. Este renglón representaba sólo el $4.22 \%$ de la población masculina de 12 y más años en 1950, en comparación con el $13.22 \%$ en 1970 (este aumento es aún mayor que el de los "estudiantes", la tercera y última categoría de inactivos: $7.75 \%$ de la población de 12 y más años en 1950, en comparación con el 13.82\% en 1970)..$^{17}$ Debe mencionarse que los 1932935 inactivos clasificados en "otros" en 1970 representaban por lo menos el 7\% de la población de 12 y más años en las edades comprendidas entre los 20 y 59 años (distribución por grupos quinquenales). Esta cifra se elevaba por lo menos al $14 \%$ en los grupos menores de 20 y mayores de 60 años. Sería deseable no sólo mencionar que esta categoría ha crecido demasiado, sino especificar cuáles de los renglones que la constituyen son responsables de este aumento. ${ }^{18}$ Por desgracia, es imposible profundizar más en este aspecto ya que nunca se ha publicado dicha información en los censos mexicanos.

El análisis anterior muestra de manera clara que los criterios de clasificación de inactivos, y por deducción también los de activos, se modificaron sustantivamente de 1950 a 1970 , aunque no exista constancia escrita de ello. A pesar de que no se cuente con la información necesaria para cuantificar la magnitud de este efecto sobre el decenso de los niveles de participación, podría sin duda afirmarse que con los criterios de 1970 se subestimó el número total de activos con respecto a 1950. Esto no quiere decir que un censo sea mejor que el otro, sino más bien que existen problemas de comparación de un caso con respecto al otro. Por supuesto que este razonamiento es extensivo a la información del año anterior al censo de 1970 , con respecto a la de 1950 , la cual se analiza en la siguiente sección, aunque por definición del tiempo de referencia de un año la subestimación posiblemente fue menor. ${ }^{\mathbf{1 9}}$

Los problemas de comparabilidad de la información femenina no se presentan de manera tan clara como en el caso anterior. Como se recordará, la tendencia de las tasas es ascendente, por lo que aquí habría

17 Información obtenida a partir del cuadro 9 del "Resumen General" del VII censo y del cuadro 32 del "Resumen General" del $I X$ censo.

18 Esta categoría, como su nombre lo indica, es más bien residual. Aquí se incluyen los encarcelados, las personas que viven en conventos o dedicadas al cuidado de enfermos, ancianos, necesitados o menores, siempre que no reciban por ello pago alguno. También se clasifican en este grupo a los que viven de sus rentas o pensiones, $\mathrm{y}$ a todas las demás personas no incluidas en los otros grupos. Cabe mencionar aquí que en los censos mexicanos se emplea la definición internacional de desocupado: personas que no tienen trabajo remunerado, pero que declaran realizar alguna actividad para encontrarlo. Si este no fuese el caso, la persona se consideraría inactiva y quedaría también incluida bajo la categoría "otros".

19 La PEA de 1969 es un poco mayor que la de la semana anterior (233 552 personas). 
que analizar la magintud de los descensos en las categorías de inactivas. De hecho, la única categoría cuya participación en el período desciende, es la de "quehaceres domésticos" (de $\mathbf{8 6 . 9 4 \% ~ d e ~ l a ~ p o b l a c i o ́ n ~ f e m e n i n a ~}$ de 12 y más años en 1950 a $82.39 \%$ en 1970). Se podría especular que este descenso es demasiado modesto, aunque en el caso de esta categoría cualquier planteamiento de esta naturaleza sería un poco aventurado, ya que no se puede controlar el número de mujeres que se encuentran prácticamente desocupadas, o trabajando a tiempo parcial, y se declaran en cambio como amas de casa. El análisis de las otras dos categorías de inactivas (estudiantes y otras) tal vez resulte un poco más útil para los fines aquí propuestos.

Las estudiantes casi duplican su participación en el período (de 5.33 a $10.15 \%$ de la población femenina de 12 y más años), lo cual habla en favor del sistema de educación mexicano; la categoría "otras", paradógicamente, también aumenta de $1.17 \%$ en 1950 a $2.57 \%$ en 1970 . Este último hallazgo permite plantear también en este caso la posibilidad de una subestimación de las inactivas en 1970 con respecto a 1950.

Razonando de esta manera se podría suponer que la participación femenina en realidad ha estado creciendo aceleradamente, en forma especial en los grupos de edad menores de 30 años. A pesar de que se necesita un estudio más profundo para llegar a comprobar esta hipótesis, existen ya algunos indicios de que la participación femenina está cambiando muy rápidamente. Por ejemplo, las tasas femeninas de la "semana anterior al censo" en 1970 (véase la gráfica 1) son superiores en todos los casos a las calculadas con base en la información de 1969.

Conforme al análisis anterior se puede concluir sin lugar a dudas que la información que se está manejando presenta problemas de comparabilidad. Sin embargo, aun para el caso de la información masculina, donde estos problemas se detectan con más claridad, sería aventurado afirmar que en ello radica la totalidad de la explicación de las tendencias observadas. Más bien la falta de comparabilidad de la información es un elemento más que debe tomarse en consideración, desafortunadamente no cuantificable, y cuya influencia aparece más clara en la tercera y última sección.

\section{Desarkollo económico y Variaciones en los Niveles de PARTICIPACIÓN}

Parece aceptarse comúnmente que un descenso en la participación masculina de la magnitud antes observada sería un resultado directo de un aumento en el nivel de desarrollo económico. Revisemos rápidamente la evidencia que existe al respecto. En un estudio sobre el particular realizado a nivel mundial por Naciones Unidas ${ }^{20}$ se calcularon, para

20 Naciones Unidas, Age Structure and Labour Supply, Actuaciones de la Conferencia Mundial de Población, 1954. Citado por Elizaga y Mellón, Aspectos demográficos de la mano de obra en América Latina, CELADE, 1971, pp. 51-60. 
alrededor de 1950, las tasas específicas de actividad masculina para varios países subdesarrollados (60\% o más de la PEA empleada en la agricultura), semidesarrollados (de 30 a $59 \%$ de la PEA empleada en la agricultura) e industrializados (menos del $30 \%$ de la PEA empleada en la agricultura). Del examen de los resultados se obtuvieron las siguientes conclusiones:

1) Las tasas de actividad masculina en edades marginales (menos de 20 y 55 y más) disminuyen sensiblemente al elevarse el nivel de desarrollo económico;

2) Al pasar del primer estado (países subdesarrollados) al segundo escalón (países semidesarrollados), la reducción se produce en las edades juveniles, en especial en el caso de los niños (menos de 15 años), y arriba de los 65 años;

3) Al pasar del segundo estado al tercero (países industrializados), la reducción más importante ocurre a partir de los 55 años de edad, o sea una extensión del proceso que antes se advertía a partir de 65 años;

4) Entre los 25 y 55 años existe una marcada analogía, cualquiera que sea el tipo de país. ${ }^{21}$

Con respecto a la participación femenina, a menudo se enfatiza que los elementos culturales que la rigen son tan diversos que imposibilitan las generalizaciones que de manera común se hacen en el caso de los hombres. En efecto, Boserup ${ }^{22}$ muestra que aun dentro del mundo subdesarrollado existen diferencias significativas en lo que toca a la participación femenina. El mundo latinoamericano, y el árabe del África del Norte y del Asia Occidental, se caracterizan por una escasa participación femenina en el campo; aunque alta en la ciudad, en el caso de América Latina, pero baja entre los árabes. En el sudeste de Asia prevalece una alta participación femenina en los dos lugares, y en África al sur del Sahara una alta participación en el campo pero baja en las ciudades (datos alrededor de 1960). Según Boserup, se combinan tres factores independientes para mantener la participación femenina a niveles bajos en el campo latinoamericano: 1) la existencia de una clase numerosa de campesinos sin tierra, 2) un sistema agrícola en el que prevalece la tracción animal, y 3) la norma cultural de confinamiento de la mujer dentro del hogar.

A su vez, esta situación tiene repercusiones en la participación femenina urbana en latinoamérica, ya que permite que gran cantidad de mujeres jóvenes busquen trabajo en las ciudades. Sin embargo, no queda claro cómo se debilita en este último caso la norma cultural del confinamiento. El hecho es que en realidad se debilita, ya que generalmente se registran proporciones de mujeres activas mucho más elevadas entre los

21 Elizaga y Mellon, ibid., p. 54.

22 Boserup, op. cit., pp. 174-193. 
trabajadores no agrícolas. ${ }^{23}$ Sería de esperar entonces que la participación femenina total aumentará a medida que se incremente el empleo no agrícola, lo cual se considera a menudo como un indicador de desarrollo económico. No obstante, Durand encontró que "las medidas de los años brutos de vida activa femenina ${ }^{24}$ no muestran una relación congruente con los niveles de desarrollo económico en los análisis transversales de 1960-1964 o de 1946-1953 (alrededor de 20 países latinoamericanos para cada período). Los cambios intercensales de los años brutos femeninos son positivos en algunos países y negativos en otros, y no siguen ningún patrón claro excepto cierta tendencia hacia la igualdad, aumentando donde han sido relativamente bajos y disminuyendo donde han sido altos. Dicha tendencia también se ha registrado en otros estudios. ${ }^{25}$ Puede observarse entonces que México no parece estar fuera del ámbito latinoamericano en lo que a la participación femenina se refiere. Sin embargo, la argumentación anterior parece más bien llevarnos a la conclusión de que los demás países de este continente comparten con México los problemas de definición analizados antes.

Cuando los autores mencionados establecen las relaciones existentes entre los diversos indicadores de desarrollo económico y los niveles de participación masculinos y femeninos, hemos visto cómo las diferencias de participación entre el campo y la ciudad, o más bien entre trabajadores agrícolas y no agrícolas parecen ofrecer un primer punto de apoyo para nuestro análisis. Veamos cómo han sido interpretadas estas diferencias en el caso más claro de la participación masculina.

Lo que generalmente se hace es enumerar algunas de las diferencias entre las áreas urbanas y las áreas rurales (consideradas como los lugares donde se ubican la industria moderna y la agricultura, respectivamente) que posiblemente tengan repercusión sobre las variaciones en la participación antes señalada: por ejemplo: 1) existen mayores demandas de calificación y eficiencia en la industria moderna que en la agricultura, y por lo tanto menores oportunidades de que niños $u$ hombres de avanzada edad permanezcan dentro de la actividad (recuérdese la importancia de estas edades en el descenso de los niveles de participación); 2) Los avances en el sistema educativo se consolidan primero en las áreas urbanas; es aquí donde cada vez hay mayor número de escuelas y de facilidades para obtener una educación formal en general. Por lo tanto, cuando aumenta la importancia relativa de estas áreas, el peso de la población escolar es mayor, de modo que los niveles de participación descienden; 3) Es en las áreas urbanas donde comienzan a generalizarse primero los sistemas de seguridad social y las pensiones en empresas particulares. Esto hace que los hombres de mayor edad se aparten de la actividad a

23 Durand, op. cit., cuadro 1, pp. 80-81.

24 Los años brutos de vida activa son probablemente la medida más refinada del nivel de participación; a través de dicha medida se elimina por completo el efecto de la estructura por edad.

25 Durand, op. cit., pp. 81-82. Véase C. E. V. Leser, "Trends in Women's Work Participation", Population Studies, noviembre de 1968. 
edades más tempranas, y que por lo tanto descienda el nivel de participación, a medida que aumenta la importancia de estas áreas urbanas.

Este esquema de "actividad diferencial campo-ciudad" ampliamente utilizado en demografía, está basado en una concepción de la sociedad bajo la cual se supone que los países subdesarrollados se encuentran en transición, de una sociedad tradicional a una moderna, transición caracterizada por una sucesión de momentos en los cuales se superponen elementos pertenecientes a estos distintos tipos de sociedad. La "sociedad tradicional" está basada en una economía de subsistencia, prácticamente agrícola, y la "sociedad moderna" se ejemplifica mediante un modelo idealizado de la sociedad urbano-industrial de los países capitalistas desarrollados. En el polo tradicional existe muy poca diferenciación de funciones, se opera a bajos niveles de eficiencia, y las acciones prescriptivas predominan sobre las electivas. (Recuérdense los factores que se consideraron importantes en la interpretación de los altos niveles de participación en el campo: oportunidades de empleo para trabajadores en las edades marginales, bajos niveles de escolaridad y menor beneficio por parte de los sistemas de seguridad social y de pensiones.) Por el contrario, en el polo moderno (urbano-industrial) la división del trabajo se somete al principio de la eficiencia, se diferencian las funciones y predominan las acciones electivas sobre las prescriptivas. (Como se recordará, el descenso en los niveles de participación que se daba más rápidamente en estas áreas se considera resultante de la mayor complejidad en la organización del trabajo a fin de operar al máximo nivel de eficiencia. Dentro de este contexto, los sistemas de seguridad social y la asistencia escolar generalizada, factores considerados también como importantes en el descenso de los niveles de participación, formarían a su vez parte de la diferenciación de funciones e instituciones que tiene lugar en una sociedad moderna.)

La utilización de este aparato conceptual pone en evidencia la poca adaptación de los estudios de PEA a la realidad del mundo subdesarrollado. A nuestro modo de ver, este discurso téorico no aclara de manera satisfactoria la realidad de nuestro cambio social, y por lo tanto tampoco el descenso en los niveles de participación. ${ }^{26}$ En primer lugar, no explica, sólo describe; y en segundo, la descripción no corresponde a nuestro caso particular, ni tampoco a muchos otros. Veamos en detalle este segundo punto.

Al presentar al campo y a la ciudad como polos antagónicos, se supone que las características de las actividades que allí se desarrollan (agrícolas-no-agrícolas) afectan a los niveles de participación de manera

26 Este no sería el lugar indicado para profundizar en estos planteamientos. Más bien analizaremos las implicaciones inmediatas del mencionado enfoque teórico para los fines específicos del trabajo. Para mayor información al respecto, véase Neide Patarra y María Coleta F. A. de Oliveira, "Anotaciones críticas sobre los estudios de fecundidad", en Reproducción de la población y desarrollo, clacso. También, Orlandina de Oliveira y Claudio Stern, "Notas acerca de la teoría de las migraciones. Aspectos Sociológicos”, en Migración y Desarrollo, cLAcso, 1972. 
exactamente opuesta. Las actividades no-agrícolas, sin embargo, son extremadamente heterogéneas; los sectores secundarios y terciarios, ${ }^{27}$ al menos en los países subdesarrollados, presentan características muy distintas. El acelerado crecimiento del segundo sector, con respecto al primero, en el caso de América Latina, se ha venido considerando como una muestra importante de los desajustes internos a que se ven sujetos los países "capitalistas dependientes". ${ }^{28}$ Veamos la evidencia que existe en nuestro caso, su significado, y cómo esto contribuye a cambiar el panorama presentado al describir el descenso de los niveles de participación.

Kurnets, en uno de los trabajos básicos sobre las transformaciones de la estructura ocupacional que acompañan al desarrollo, ${ }^{29}$ señaló un hecho sobre el que se ha especulado bastante en estudios posteriores. Nos referimos a la excesiva terciarización del mundo subdesarrollado. En efecto, Cardoso y Reyna, en un estudio sobre el particular en América Latina, afirman que "mientras en los países de desarrollo originario, el sector primario de la economía disminuyó en beneficio del secundario, en cambio, en América Latina la expansión rápida del terciario sucede desde el comienzo del proceso de industrialización. Así, lo que fue un efecto tardío de la industrialización en Europa Occidental y Estados Unidos - la formación de amplios sectores terciarios- se manifiesta desde los comienzos mismos del proceso de industrialización en América Latina". ${ }^{30}$

Un aspecto fundamental que debe tomarse en cuenta en cualquier análisis detallado que se haga al respecto, es el de la gran heterogeneidad de la estructura interna del sector terciario. Este engloba actividades que son necesariamente complementarias de la producción de bienes - comercio, transportes y comunicaciones- así como servicios colectivos y servicios de escasa productividad, casi siempre englobados bajo servicios individuales.

Por lo general se sostiene que las del terciario son las actividades de más baja productividad, ya que éste es un sector de refugio, al cual se incorpora gran parte de la población activa desplazada del sector primario, e incapaz de ser absorbida por el secundario. En el estudio de Cardoso y Reyna, por ejemplo, se encontró que el sector "servicios" (guber-

27 Siguiendo la clasificación de Colin Clark, el sector primario incluye la rama de agricultura, ganadería, silvicultura, caza y pesca; el sector secundario las ramas de industrias extractivas y de transformación, la construcción y la generación, transmisión y distribución de energía eléctrica; por último, el sector terciario comprende las ramas de comercio, transportes y la de servicios y gobierno. Véase, Colin Clark. The Conditions of Economic Progress, Londres, Macmillan Co., 1957.

28 Véase, Castells M. “L'urbanization dependante en Amerique Latine”, Espaces et Societes, julio de 1971, Núm. 3, pp. 5-23.

29 Simon Kuznets, "Quantitative Aspects of the Economic Growth of Nations", Economic Development and Cultural Change, Vol. 5, 1956-1957 (en especial la segunda parte sobre, "Industrial Distribution of National Product and Labor Force").

30 Fernando H. Cardoso y J. Luis Reyna, "Industrialización, estructura ocupacional y estratificación social en América Latina", en Cuestiones de Sociología del Desarrollo, Santiago de Chile, Editorial Universitaria, 1968, pp. 68-105. 
namentales, privados, y ocupaciones no especificadas) en Argentina, Chile, México y Venezuela, en el decenio 1950-1960, representaba la proporción mayor de población activa dentro del terciario, y la que crecía más rápidamente (comparada con comercio, finanzas, transportes y comunicaciones). Según los autores, en estudios realizados en Chile y Perú se ha encontrado hasta un $25 \%$ de "desocupación disfrazada" en los subgrupos de "servicios varios y actividades no especificadas".

Sin embargo, en un artículo más reciente, Miller presenta evidencia de que está ocurriendo precisamente lo contrario de lo expuesto antes ${ }^{31}$ (conviene no obstante aclarar de antemano que los dos estudios no son estrictamente comparables, ya que incluyen diferentes países de América Latina, y las subdivisiones de los servicios que se emplean son distintas. Miller utiliza: servicios del gobierno, de la comunidad y personales). La autora sostiene que los aumentos en la participación de los servicios y el comercio en el empleo no agrícola son "generales pero moderados". Además afirma que: "en los pocos países para los cuales se cuenta con información de dos períodos (Guyana, 1946-1960; Venezuela, 1950-1961; Trinidad-Tobago, 1946-1960; Puerto Rico, 1950-1960) sobre los componentes del sector servicios, los servicios personales, tal vez los menos productivos, disminuyen en cuanto a su importancia relativa. "Aquí también las series presentes no tienden a dar sustancia a la hipótesis de que el empleo no-agrícola se concentra cada vez más en las industrias menos productivas", ${ }^{32}$ Un aspecto adicional que muestra el estudio de Miller es que, dentro de los servicios personales, también bajó la participación del servicio doméstico para todos los casos estudiados.

Para el caso de México, Unikel y Torres ${ }^{33}$ afirman que, "en el decenio 1940-1950, la PEA del sector secundario creció a razón de $6.7 \%$ anual y la del terciario a $5.1 \%$; en el decenio siguiente los dos sectores registraron la misma tasa $(3.9 \%)$. En consecuencia, la importancia relativa del sector secundario aumentó durante el período: la relación entre trabajadores ocupados en el sector terciario y el secundario bajó de 1.7 en 1940 a 1.4 en 1960, tendencia que se supondría positiva en el caso de que comprobara que la contracción relativa ocurrió en los estratos de menor productividad". ${ }^{34}$ No obstante, los autores establecen que, "las cifras anteriores no dan una idea de la evolución interna de cada sector y es incluso posible que pese a que la industrialización ha sido más rápida que la terciarización, hayan crecido más aprisa los estratos de baja productividad del sector terciario y haya aumentado el "desempleo disfrazado" en este sector en mayor medida que en otros. Sin embargo,

31 Ann Miller, "Algunas características de la estructura industrial del empleo en países latinoamericanos", Conferencia Regional Latinoamericana de Población, Actas 2, El Colegio de México, 1972, pp. 83-91.

32 Miller, ibid., p. 90.

33 L. Unikel y F. Torres, "La población económicamente activa en México y sus principales ciudades, 1940-1960", Demografía y Economía, El Colegio de México, Vol. IV, Núm. 1, 1970, pp. 1-42.

34 Ibid., p. 10. 
MÉxico: Población económicamente activa de MICA, ${ }^{a}$ AMBOS SE

(Porci

\begin{tabular}{|c|c|c|}
\hline Rame de aotividad & $\begin{array}{c}1950^{2} \\
\text { (1) }\end{array}$ & $\begin{array}{l}1970 \\
(2)\end{array}$ \\
\hline $\begin{array}{l}\text { Agrioul tura, ganaderia, silvioul- } \\
\text { tura, oase y pesoa } \\
\text { Primario }\end{array}$ & $\begin{array}{l}58.15 \\
58.15\end{array}$ & $\begin{array}{l}39.40 \\
39.40\end{array}$ \\
\hline $\begin{array}{l}\text { Indus trias axtraotivas (petr6leo, } \\
\text { gas natural, explotaoión de mi- } \\
\text { nas y oanteras) }\end{array}$ & 1.26 & 1.39 \\
\hline Industrias de transformaoion & 11.74 & 16.74 \\
\hline Construooi 6n & 2.75 & 4.40 \\
\hline $\begin{array}{c}\text { Genorao16n, trensmision y distri- } \\
\text { buo16n de onergia ol ootrioa } \\
\text { Seoundario }\end{array}$ & $\begin{array}{r}0.30 \\
16.05\end{array}$ & $\begin{array}{r}0.41 \\
22.94\end{array}$ \\
\hline Comerolo & 8.24 & 9.24 \\
\hline Tranaportos & 2.56 & 2.84 \\
\hline Sorvioion I sobierno & 10.61 & 19.80 \\
\hline Teroiario & 21.41 & 31.88 \\
\hline Insurioien temonte espooifiosdee & 4.39 & 5.78 \\
\hline Sotal & 100.00 & 100.00 \\
\hline
\end{tabular}

Fuente: VII y IX Censos Generales de Población.

a La clasificación por rama de actividad no es estric apéndice 3 se muestra que existen varios ajustes $p$ por subgrupos de actividad económica. Sin embargo, pos de edad en ninguno de los censos. Como éste es cipación que se realiza más adelante, se optó por usar fue publicada, de modo que los datos de los dife últimas dos columnas de este cuadro se utiliza la torsiones que introduce el uso de los datos no ajus mación sobre subgrupos de actividad económica de Y Economía, Vol. VII, número 2, 1973, pp. 249-264.

b En 1950 la información por ramas de actividad no

c La información de 1950 sobre ramas de actividad de trabajo no incluye a los desocupados por 13 sema dad, hubo que distribuir a estos desocupados entre 33-A del Resumen General del VIII Censo.

d Información ajustada, véase Brígida García, loc. cit. 
ro 7

12 AÑOS Y MÁS POR RAMAS DE ACTIVIDAD ECONÓXos, $1950-1970^{\mathrm{b}}$

entos)

\begin{tabular}{|c|c|c|}
\hline $\begin{array}{c}\text { Cambio } \\
\text { poroon tual } \\
\text { (3) }\end{array}$ & $\begin{array}{l}\text { Cambio } \\
\text { poroentual } \\
\text { (4) }\end{array}$ & $\begin{array}{l}\text { Partioipacion on el in } \\
\text { oromonto do la pend } \\
\text { (5) }\end{array}$ \\
\hline-32.25 & -32.25 & 5.46 \\
\hline-32.25 & -32.25 & 5.46 \\
\hline 10.31 & 10.31 & 1.62 \\
\hline 42.58 & 58.17 & 30.94 \\
\hline 60.00 & 60.00 & 7.40 \\
\hline 36.66 & 57.69 & 0.67 \\
\hline 42.93 & 54.72 & 40.63 \\
\hline 12.13 & 16.81 & 11.64 \\
\hline 10.93 & 25.11 & 3.89 \\
\hline 86.61 & 59.44 & 30.11 \\
\hline 48.90 & 40.09 & 45.64 \\
\hline 31.66 & 31.66 & 8.27 \\
\hline- & - & 100.00 \\
\hline
\end{tabular}

tamente comparable en los dos años considerados. En el osibles en el caso de que se desglose la información la información por subgrupos no fue tabulada por gruun dato esencial para el análisis de niveles de partila información por ramas de actividad, tal y como rentes cuadros fuesen comparables. No obstante, en las información ajustada, a fin de poder apreciar las distados (véase Brígida García, "Comparación de la inforlos Censos de Población de 1950 a 1970", Demografía

fue tabulada para cada sexo por separado.

corresponde a fuerza de trabajo y no a PEA (la fuerza nas y más). Para obtener la PEA por ramas de activilas diversas ramas según la información del cuadro 
las elaboraciones estadísticas de que se dispone a este respecto son todavía burdas (véanse, por ejemplo, las elaboraciones sobre el subempleo en América Latina que aparecen en el estudio de ILPES-CELADE, Elementos para la elaboración de una política de desarrollo con integración para América Latina). Además, en los tres años analizados, el producto por trabajador ha sido superior en el sector terciario. Mientras no se disponga de datos más detallados, la diferencia del producto sectorial por trabajador no respalda la hipótesis de estratos mayores de baja productividad en el sector terciario, a pesar de la presencia de tales grupos socioeconómicos, cuya magnitud real y características se desconocen".35

Con respecto al período 1950-1970, en su conjunto, en el cuadro 7 se presentan los cambios efectuados en el crecimiento de la PEA a nivel de ramas de actividad económica. No obstante las múltiples limitaciones que esta información ofrece (véanse las notas de dicho cuadro) permite analizar varios puntos importantes. Si observamos la columna 2, podremos notar que el sector primario continúa siendo el que absorbe más mano de obra, aunque ya sólo se sitúe aquí el $39.40 \%$ de la PEA en 1970, comparado con el 58.15 en 1950. Le siguen en importancia el sector terciario - $31.88 \%$ de la PEA en 1970- donde se destaca la rama de servicios y gobierno, y luego el sector secundario $-22.94 \%$ de la PEA en 1970 - donde la industria de transformación ocupa el primer lugar en absorción de mano de obra. Por último, habría que anotar también que las actividades insuficientemente especificadas de hecho aumentaron su participación en 1970.

Con respecto al cambio observado en el período (columna 3), es importante destacar el aumento significativo de las actividades no-agrícolas en desmedro de las actividades agrícolas. Las actividades terciarias dentro de las no-agrícolas parecen haber experimentado el más alto crecimiento, destacándose la rama de servicios y gobierno, que parece haberse expandido de forma más acelerada que cualquier rama tomada de manera individual. No obstante, éste es un fenómeno que en parte se deriva de la falta de comparabilidad en la información (en 1970, a diferencia de 1950, se consideró que la división del trabajo en México había evolucionado lo suficiente como para considerar a los servicios de reparación como "servicios" y no como "industria de transformación". En 1970, 236126 personas de ambos sexos se encontraban clasificadas en estos servicios; esto abulta de manera desproporcionada a los servicios de ese año en comparación con los de 1950; véase la columna 4). La información ajustada señala entonces, de una manera más clara, cómo todas las ramas del secundario, a excepción de las industrias extractivas, experimentaron un aumento significativo durante el período. ${ }^{\mathbf{3 6}}$ Las ramas

35 Ibid., pp. 11-12.

36 Debe aclararse que el ajuste realizado pudo haber introducido sesgos adicionales, ya que tuvimos que adoptar el criterio de 1950 -servicios de reparación como parte de la industria de transformación- ya que la información sobre dichos servicios sólo podía desagregarse en el caso de 1970. Lo más probable es 
del terciario, en cambio, aumentaron en forma menos marcada, a excepción de la rama servicios y gobierno. Sin embargo, hay que observar que los niveles en el sector secundario en 1950 eran mucho menores que los del sector terciario, y que este último continúa absorbiendo en 1970 un porciento mayor de mano de obra que el secundario. Por último, es importante señalar que las actividades insuficientemente especificadas experimentaron un aumento de $31.66 \%$ durante el período; éste es un hecho que también impide en parte conocer la verdadera magnitud de los cambios que han tenido lugar.

Si cbservamos ahora la columna 5 , podremos notar cuáles ramas se destacaron en la absorción del incremento de mano de obra durante el período. E1 sector terciario ocupa el primer lugar en este sentido $(45.64 \%)$ aunque seguido muy de cerca por el secundario $(40.63 \%)$. Es notoria, por otra parte, la escasa absorción de mano de obra por el sector primario de la economía $(5.46 \%)$. En cambio, dos ramas noagrícolas se destacan por su alta absorción: "industria de transformación" (30.94\%) y "servicios y gobierno" $(30.11 \%)$. Probablemente éstas sean las ramas que presentan una estructura interna más heterogénea, tal y como fue planteado en la discusión anterior con respecto a los servicios.

En lo que toca a la industria de transformación, Soza Valderrama afirma que "la heterogeneidad del sector manufacturero puede considerarse desde tres ángulos principales. En primer lugar, la producción industrial tiene diversos destinos: el consumo final, la utilización intermedia y la formación de capital, además, por supuesto, de la exportación; el sector comprende, de otra parte, una gran variedad de industrias, económica y técnicamente tan diferentes como las de elaboración de alimentos y las metalúrgicas; por último, suelen convivir en él mundos tecnológicos, económicos y sociales tan distintos como los estratos artesanales y fabriles" ${ }^{37}$ La información que manejamos no nos permite clasificar a las industrias en artesanales y fabriles, o analizar las diferencias tecnológicas que existen entre ellas. Nos permitirá en cambio investigar, tanto para esta rama como para la de "servicios y gobierno", qué tipo de industrias o servicios son responsables del aumento de mano de obra antes señalado. En el cuadro 8 se presenta la participación de las ramas de "industria de transformación" y de "servicios y gobierno", así como la de sus componentes, en el incremento de la PEA durante el período.

En lo que respecta a la industria de transformación, Soza Valderrama, en su libro antes mencionado, observa para una muestra de 29 países, que a medida que aumenta el grado de desarrollo y la industrialización, disminuye la significación relativa de las industrias de bienes de consumo no duradero, aumenta la importancia porcentual de las de bienes intermedios, y crece muy rápidamente la ponderación de las de

que este criterio no se ajuste a la realidad mexicana actual, y que por tanto, la información no nos permita apreciar el verdadero crecimiento de la industria de transformación y de los servicios.

37 Héctor Soza Valderrama, La planificación del desarrollo industrial, México, Siglo XXI, 1966, p. 21. 


\section{Cuadro 8}

MÉXico: Participación de las Ramas de "INDUStria de transformación" Y DE "SERVICIOS Y GOBIERNo" EN EL INCREMENTO DE LA PEA

(AMBos SExos, 1950-1970)

(Porcientos)

\begin{tabular}{|c|c|}
\hline Rama de actividad & $\begin{array}{l}\text { Incremento } 1950-70 \\
\text { Información ajustada }\end{array}$ \\
\hline Industrias de Transformacion & 30.94 \\
\hline $\begin{array}{l}\text { Industrias productoras de bines de consumo, } \\
\text { especialmente no duradoros, intermedios } \\
\text { relacionados b/ }\end{array}$ & 12.27 \\
\hline $\begin{array}{l}\text { Industrias más tipicamento productoras de } \\
\text { bienes intermedios } q\end{array}$ & 6.42 \\
\hline $\begin{array}{l}\text { Industrias más tipicamente productoras de } \\
\text { bienes de capital y de consumo duradero of }\end{array}$ & 10.52 \\
\hline Insufioientemente espeoificados & 1.73 \\
\hline Servioios $y$ Gobierno & 30.11 \\
\hline Servicios de producción $e /$ & 6.17 \\
\hline Servicios de consumos & 11.79 \\
\hline Servicios colectivog & 12.01 \\
\hline Insufici ent ewante especificados & 0.14 \\
\hline
\end{tabular}

Fuentes: VII y IX Censos Generales de Población.

a Véase Brigida García, loc. cit.

b Incluye alimentos, bebidas, tabaco, textiles, calzado y vestuario, cuero, madera, muebles e imprentas.

c Incuye papel, caucho, químicas, minerales no metálicas y metálicas básicas.

d Incluye productos metálicos, maquinaria eléctrica y mecánica, material de transporte y diversas. Véase Soza Valderrama, op. cit., p. 22.

c Incluye los servicios de alquiler y diversos, servicios de instituciones de crédito, auxiliares de crédito e instituciones de seguros y los servicios telefónicos, de radiocomunicación y telex.

if Incluye los servicios de esparcimiento, los de alojamiento temporal, los de preparación y venta de alimentos y bebidas, los servicios de profesionales, y los servicios domésticos.

g Incluye los servicios de enseñanza, los de asistencia médica y social, las organizaciones religiosas y la división Gobierno. Véase Paul Singer, op. cit., p. 28.

manufactura de capital y de consumo duradero (la importancia relativa de cada industria está medida en términos de valor agregado de la producción).$^{38} \mathrm{El}$ autor aclara que esta situación tiene su origen en el comportamiento de la demanda de manufacturas y en el proceso de sustitución de importaciones. Por una parte, las elasticidades-ingresos de las distintas manufacturas varían en el sentido que muestra el crecimiento de las diferentes industrias; por la otra, las mayores dificultades técnicas y económicas para la producción de bienes intermedios y de capital, y el hecho de que su demanda sea escasa en las primeras etapas del desarrollo, explica que las industrias de bienes de consumo se instalen antes. La sustitución de bienes de consumo hace aparente la demanda de bienes intermedios, que ya venían en gran parte incorporados o encubiertos en

38 Ibid, pp. 21-30. 
las anteriores importaciones de dichos bienes de consumo; la demanda de bienes de capital aparece en términos más significativos una vez que se comienzan a instalar las primeras industrias sustitutivas. Es importante enfatizar que el autor aclara que "este esquema de sustitución de importaciones, de validez histórica para la mayoría de países en desarrollo, no es necesariamente el más adecuado...., pues hay buenas razones para pensar en la conveniencia de un desarrollo industrial mejor integrado".39

El panorama se complica cuando buscamos explicar las tendencias en la absorción de mano de obra en las distintas industrias de transformación, debido a las diferencias tecnológicas involucradas. En el caso de México podemos observar que las industrias productoras de bienes de consumo, las que ocupan más trabajadores por unidad de capital, también ocuparon el primer lugar en absorción de mano de obra durante el período $(12.27 \%)$. No obstante, las industrias productoras de bienes de capital y de consumo duradero, las que en contraste ocupan menos trabajadores por unidad de capital, absorbieron también un importante contingente de mano de obra durante 1950-1970 (10.52\%). Esto nos indicaría que México se encuentra en una etapa avanzada del proceso de sustitución de importaciones, en el caso de que éste se aceptara como válido. Sin embargo, hay que aclarar que la cifra de las industrias de bienes de capital se encuentra desproporcionalmente abultada; aquí se incluyen todos los servicios de reparación, los cuales obviamente no forman parte de una última etapa en el proceso de sustitución antes mencionado (véase la nota 36 ).

En lo que concierne a la rama de "servicios y gobierno", los datos indican que la situación es mucho más compleja de lo que la discusión anterior parecía señalar. Por una parte, hemos visto que el proceso de industrialización mexicana ha sido muy dinámico durante el período estudiado (además de la industria de transformación, la construcción y la energía eléctrica también experimentaron aumentos significativos -véase el cuadro 7-; por lo tanto, los servicios que necesariamente acompañan a este proceso (servicios de producción), así como aquellos destinados a satisfacer las demandas de una población creciente (servicios colectivos) han absorbido también una cantidad nada desdeñable de mano de obra $(6.17$ y $12.01 \%$, respectivamente; obsérvese también en este sentido el crecimiento del comercio y de los transportes, actividades también suplementarias del proceso de industrialización - véase el cuadro 7). Por otra parte, aquellos servicios denominados de baja productividad (de consu$\mathrm{mo}$ ), y donde se afirma que prevalece el desempleo disfrazado, ocupan también un lugar importante en cuanto a absorción de mano de obra se refiere $(11.79 \%)$. No obstante, es importante destacar que dentro de dichos servicios los llamados domésticos sólo aumentaron $12 \%$ durante el período, en comparación con $66 \%$ de los de esparcimiento, $80 \%$ los de alojamiento temporal, y $111 \%$ los de preparación y venta de alimentos $\mathrm{y}$ bebidas (es probable que los servicios que han crecido más sean

39 Ibid, p. 26. 
también de muy baja productividad; sin embargo, faltan estudios específicos a este respecto). En conclusión, aunque el proceso de industrialización mexicana, y sus servicios correspondientes, dan muestras de avance a paso acelerado, el estudio más global de la estructura ocupacional señala además la existencia de procesos paralelos que ponen a su vez de relieve la insuficiencia de dicho crecimiento para absorber la mano de obra disponible.

Como se ha podido observar, la dinámica del sector no-agrícola mexicano, descrita con algún detalle, es muy compleja y se encuentra bastante alejada de la situación expuesta al inicio de esta sección. Con estos nuevos elementos, retomemos ahora el estudio de la relación entre el desarrollo económico y los niveles de participación.

En el cuadro 9 se presentan los cambios porcentuales en las tasas específicas de actividad, ahora clasificadas por ramas de actividad económica para 1950-1970. La imposibilidad de analizar esta información para cada sexo por separado (véase la nota $b$ del cuadro 9) limita en parte el alcance de las siguientes conclusiones, pero permite al menos ampliar el panorama presentado en la sección anterior. Varios puntos importantes merecen ser destacados:

1. Las cifras totales muestran un descenso significativo en la participación de todos los grupos de edad, a excepción de los que van de los 20 a los 44 años; por el contrario, las tasas estimadas para 1950 señalaban un descenso importante en la participación de todos los grupos de edad para el período 1950-1970. De lo anterior se deduce que dichas tasas de hecho sobrestiman el nivel de participación en dicha fecha con respecto a la información censal utilizada para elaborar el cuadro 9. Aunque este hallazgo no modifica sustancialmente las conclusiones de este trabajo, ${ }^{40}$ puede considerarse al menos como un subproducto importante del mismo.

2. Puede apreciarse que el descenso de las tasas de participación en el sector agrícola juega un papel importante en la interpretación del descenso global. El cambio en la participación del primero y el último grupo de edad es negativo en los tres sectores de la economía; la participación de las demás edades, en cambio, sólo desciende en el caso del sector primario. El aumento observado en dichas edades en el caso del secundario y del terciario no alcanza a contrarrestar esta tendencia descendente en el sector primario, y el resultado global es negativo para los grupos 15-19 y 45-64, y de muy escaso aumento para el de 20-24 $\mathrm{y}$ el de $25-64$ años (2.94 y $0.24 \%$ de aumento respectivamente). Visto

40 Se observará que las tasas estimadas para 1950 fueron utilizadas casi exclusivamente para fines de la tipificación. En este respecto se demostró -véase el apéndice 2 de la versión ampliada- que el resultado principal de este ejercicio estadístico (la escasa influencia de la estructura por edad sobre los cambios de la TRA) no variaba sustancialmente cuando se usaban en dicho ejercicio las tasas específicas gruesas calculadas a partir de la información censal que se presentan en el cuadro 7, en vez de las tasas estimadas de los cuadros 3 y 4. 


\section{Cuadro 9}

México: Cambios porcentuales en las tasas específicas de actividad POR RAMAS DE ACTIVIDAD ECONÓMICA, ${ }^{a}$ AMBOS SEXOS, ${ }^{b}$ 1950-1970 c

\begin{tabular}{|c|c|c|c|c|c|c|}
\hline & \multicolumn{6}{|c|}{ Grupos de edad } \\
\hline & $12-14$ & $15-19$ & $20-24$ & $25-44$ & $45-64$ & $65 \mathrm{y}$ más \\
\hline $\begin{array}{l}\text { Total } \\
\text { A Ericultura, ganaderfa } \\
\text { silvioul tura, caza y } \\
\text { pesca }\end{array}$ & $-67 \cdot 45$ & -21.80 & -39.57 & -31.17 & -5.30 & -56.81 \\
\hline Primario & -77.59 & -51.79 & -39.57 & -31.17 & -27.47 & -33.69 \\
\hline $\begin{array}{l}\text { Industrias extraotivas } \\
\text { Industrias de transfor- }\end{array}$ & -83.34 & -8.34 & -6.06 & 15.58 & 14.28 & -18.19 \\
\hline $\begin{array}{l}\text { mación } \\
\text { Construcoión } \\
\text { Generaoion, transmisión y } \\
\text { distribuoion de energfa }\end{array}$ & $\begin{array}{l}-47 \cdot 37 \\
-62 \cdot 17\end{array}$ & $\begin{array}{l}16.81 \\
26.66\end{array}$ & $\begin{array}{l}57.84 \\
50.67\end{array}$ & $\begin{array}{l}37.55 \\
62.13\end{array}$ & $\begin{array}{l}18.86 \\
54.73\end{array}$ & $\begin{array}{l}-17.47 \\
-1.09\end{array}$ \\
\hline el6otrica & - & -1.25 & 25.00 & 45.00 & 16.66 & $-55 \cdot 56$ \\
\hline Seoundario & -52.04 & 16.66 & 52.28 & 40.27 & 24.87 & $-15 \cdot 40$ \\
\hline $\begin{array}{l}\text { Comercio } \\
\text { Transportes } \\
\text { Servicios y gobierno }\end{array}$ & $\begin{array}{c}-54.84 \\
14.00\end{array}$ & $\begin{array}{r}16.03 \\
-32.26 \\
50.10\end{array}$ & $\begin{array}{r}39.09 \\
-19.18 \\
76.41\end{array}$ & $\begin{array}{r}2.17 \\
12.22 \\
42.95\end{array}$ & $\begin{array}{r}-8.49 \\
16.20 \\
24.29\end{array}$ & $\begin{array}{l}-18.76 \\
-1.79 \\
-5.45\end{array}$ \\
\hline Terciario & -20.07 & 31.82 & 51.24 & 23.62 & 8.77 & $-12 \cdot 17$ \\
\hline $\begin{array}{l}\text { Insuficientemente espe- } \\
\text { oificada }\end{array}$ & 13.56 & 16.16 & 11.49 & 16.18 & 37.27 & 36.44 \\
\hline
\end{tabular}

Fuentes: VII y IX Censos Generales de Población.

a Estos datos corresponden a la información no ajustada; vease nota a, cuadro 7 .

b Esta información no se tabuló por sexos en el caso de 1950.

c Para obtener la PEA de 1950 por edad y ramas de actividad hubo que distribuir a los desocupados por 13 semanas y más entre las diversas ramas, según la información del cuadro 33-A del Resumen General del VII Censo; véase la nota c, cuadro 7 .

de esta manera, resulta ahora más claro cómo el descenso en los niveles de participación ha sido causado más bien por una combinación de los factores hasta aquí analizados. Sin duda, parte del descenso en el primero y el último grupo de edad ha sido originado por los factores de modernización antes mencionados; aquí habría que mencionar el importante papel jugado por el sector primario de la economía, en oposición al del sector terciario, por ejemplo. No habría que olvidar, sin embargo, que el descenso en el primer caso fue mayor que en el último, probablemente porque se parte de tasas más altas en 1950, en lo que al sector primario se refiere. Ahora, resulta casi imposible que el cambio observado en los grupos de edad centrales pueda tener su origen principial en los factores de modernización antes mencionados, ya que esto colocaría a México en un estadio superior a su actual desarrollo. Aquí es donde se hacen más evidentes los problemas de comparabilidad de la información estudiados en la sección anterior. La información aquí analizada presenta no obstante la ventaja de permitir deducir en alguna medida el origen de la gran cantidad de inactivos que presenta la información de 1970. Con base en los datos del cuadro 9, es posible afirmar que se están captando de alguna manera como inactivos personas que en realidad no encuentran ubicación en el sector primario y que no han podido incor- 
porarse al secundario y terciario, por muy dinámica que haya sido la evolución de estos sectores en el período.

3. Tal y como se suponía, los cambios en el sector secundario ejercieron una influencia distinta sobre los niveles de participación que las transformaciones operadas en el terciario. El sentido en que cambian las tasas es el mismo para los dos sectores, pero la magnitud de dicho cambio varía en cada caso:

a) El descenso para la primera y la última edad es mucho menor en el caso del terciario que en el del secundario. Inclusive, el cambio en la edad 12-14 es positivo para la rama de servicios y gobierno (14\% de aumento). Esto nos indica que el proceso de modernización, que en cierta medida ha tomado lugar en la actividad no-agrícola, dista mucho de ser un proceso uniforme. Aun dentro de los sectores existen diferencias importantes; por ejemplo, la rama de industria de transformación, donde se supone que imperan en mayor medida las normas de eficiencia de una sociedad moderna, es la actividad donde se observa una menor disminución en la edad 12-14 dentro del secundario $(47.37 \%)$. También el descenso en la última edad es uno de los menores dentro del sector. Esta situación podría tener su origen en el hecho de que las industrias de bienes de consumo son las que absorben una proporción mayor de mano de obra dentro de la rama, además de la presencia de la producción artesanal, la cual puede suponerse importante. Es factible que los niveles de calificación sean menores en la industria de bienes de consumo que en las de bienes intermedios y de capital, así como en la producción artesanal comparada con la fabril. Por lo que se refiere al sector terciario, el aumento de la participación del grupo 12-14 en la rama de servicios y gobierno, aun cuando esta rama se encuentra abultada en forma desproporcionada en la información sin ajustar, es un hallazgo verdaderamente importante. A nuestro modo de ver es un elemento adicional que reafirma el supuesto de que los empleos de baja productivi-

dad tienden a crecer dentro del sector.

b) En lo que concierne a la participación en los grupos de edad que van de los 15 a los 64 años, también existen diferencias importantes entre los sectores. En los dos casos los cambios se hacen cada vez mayores hasta llegar al grupo de edad 20-24, y luego comienzan a descender hasta que estos se hacen negativos en el grupo 65 y más. Los aumentos son menores en el terciario a partir del grupo $20-24$, pero no así en lo que respecta al grupo 15-19. En este caso el aumento en el terciario es el doble del observado en el secundario (31.82 y $16.66 \%$, respectivamente). Es decir, el sector no-agrícola incorpora cada vez más gente joven, pero el grueso del nuevo contingente de la fuerza de trabajo es absorbido por el sector terciario de la economía, y èn especial por la rama de servicios y gobierno $(50 \%$ de aumento en el grupo 15-19). He aquí un elemento adicional que sitúa en desventaja al sector terciario en lo que se refiere a niveles de calificación y supuesta 
eficiencia y que además pone en evidencia los desajustes internos del sistema económico mexicano.

\section{Consideraciones finales}

Para resumir, pueden mencionarse los resultados que se consideran más importantes en el presente trabajo, los cuales contribuyen en mayor o menor medida a aclarar el descenso de los niveles de participación de la población en la actividad económica, durante el período 1950-1970:

1) La contribución de los factores demográficos al mencionado descenso -en especial del crecimiento de la población y de los cambios consiguientes en la estructura por edad- tuvo sólo una importancia secundaria. A este respecto, el crecimiento de la población menor de doce años fue el factor más importante; dicho crecimiento explica el $31.06 \%$ del descenso total.

2) Las causas principales del descenso se atribuyeron a factores por desgracia no cuantificables, pero bastante evidentes. Hasta cierto punto se probó la existencia de un cambio en los criterios censales de 1970, por el cual sobrestimó el número de inactivos con respecto a 1950. Se pudo deducir que al menos una parte importante de esos inactivos son en realidad personas que no encuentran ubicación en el sector primario y que tampoco han podido incorporarse al secundario o al terciario, a pesar del dinamismo experimentado por estos dos sectores durante el período. Además del efecto atribuido a los cambios en las definiciones censales, se concluyó que el descenso en los primeros grupos de edad y también en los últimos se debía en parte a ciertos avances en materia social logrados durante el período, en especial mayor educación, y la instauración más generalizada de sistemas de seguridad social públicos y privados.

3) Por último, un subproducto importante de este estudio ha sido mostrar cómo los cambios en el sector secundario ejercen una influencia distinta sobre los niveles de participación que las transformaciones operadas en el terciario. En primer lugar, los descensos en los primeros grupos de edad y en los últimos son menores en el terciario que en el secundario, e inclusive el cambio es positivo en la rama de servicios y gobierno para el grupo de edad 12-14. En segundo término, se demostró que el sector no-agrícola en su totalidad incorpora cada vez más gente joven, pero que el grueso del nuevo contingente de la fuerza de trabajo es absorbido por el sector terciario de la economía, y en especial por la rama de servicio y gobierno.

Se espera que el conjunto de estos resultados al menos ponga en relieve la complejidad que reviste un descenso de la magnitud observada en los niveles de participación. No se pretende haber agotado el tema, sino más bien haber aportado algunos elementos a la dilucidación de un problema, que a menudo se acepta sólo como un mal resultante del crecimiento de la población, cuando éste es apenas uno de sus condicionantes inmediatos. 